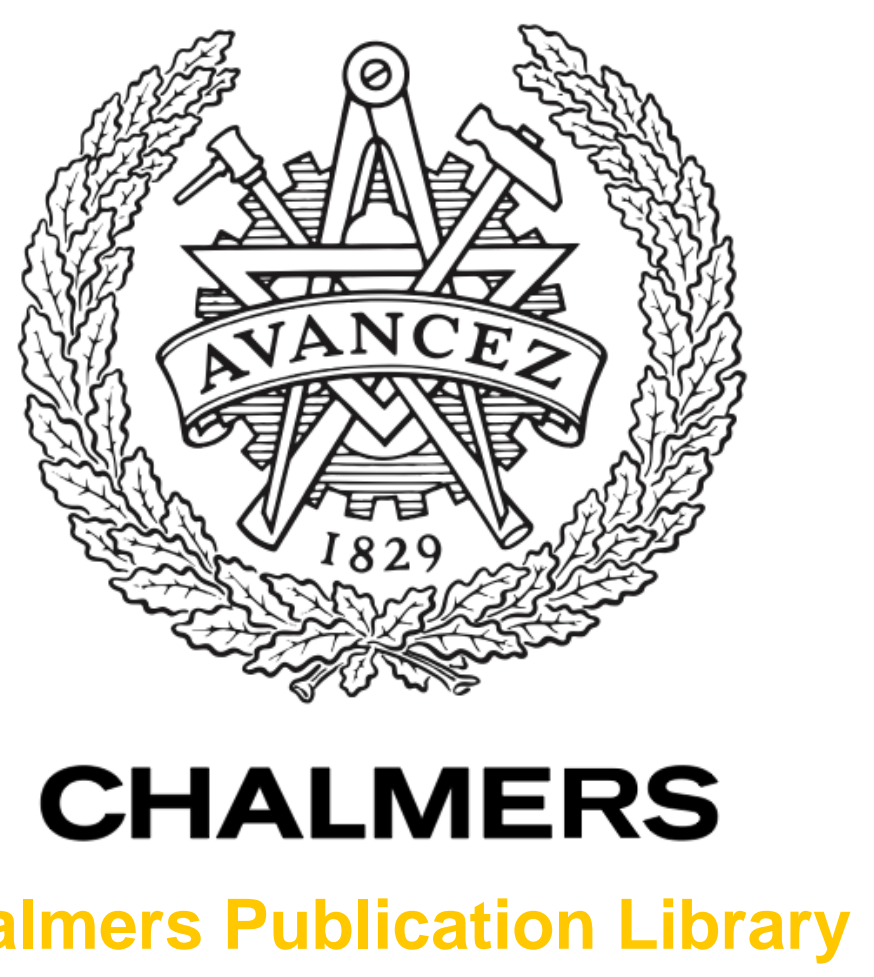

\title{
Sensor selection in magnetic tracking based on convex optimisation
}

This document has been downloaded from Chalmers Publication Library (CPL). It is the author's version of a work that was accepted for publication in:

\section{Electronics Letters (ISSN: 0013-5194)}

Citation for the published paper:

Talcoth, O. ; Rylander, T. (2013) "Sensor selection in magnetic tracking based on convex optimisation". Electronics Letters, vol. 49(1), pp. 15-16.

http://dx.doi.org/10.1049/el.2012.3030

Downloaded from: http://publications.lib.chalmers.se/publication/177444

Notice: Changes introduced as a result of publishing processes such as copy-editing and formatting may not be reflected in this document. For a definitive version of this work, please refer to the published source. Please note that access to the published version might require a subscription. 


\section{Sensor selection in magnetic tracking based on convex optimization}

\section{O. Talcoth and T. Rylander}

The performance of a magnetic tracking system is substantially influenced by the positions of its sensors. In order to optimize these sensor positions, we exploit a performance metric based on the Fisher information matrix and a convex relaxation of a sensor selection problem. Optimized sensor positions are presented for a magnetic tracking system with a planar sensor array and a measurement domain consisting of one point.

Introduction: Magnetic tracking, where the position and orientation of an object is determined by means of a quasi-magnetostatic sensor system, has numerous applications in biomedical engineering due to the transparency of the human body to low-frequency magnetic fields. For example, applications include tracking of the human eye [1], catheter tracking [2] and real-time organ positioning during radiotherapy of cancer tumors [3].

The performance of a magnetic tracking system is substantially influenced by its sensor positions. Shafrir et al. [4] used a two-step evolutionary algorithm to optimize the sensor positions of a magnetic tracking system. However, their algorithm suffers from long simulation times as "[they] had to run $10^{3}$ iterations of the tracking algorithm" for each transmitter position in order to assess the impact of measurement noise.

In this work, we follow the method in [5] and formulate an optimization problem that exploits the Fisher information matrix. In order to solve the optimization problem, we express it as a sensor selection problem that, after relaxation of the integer constraints, is turned into a convex optimization problem. Our approach assesses the impact of measurement noise without having to build statistics by running a tracking algorithm. Furthermore, the results are valid for all unbiased estimators instead of a particular tracking algorithm as in [4].

Modeling: The quasi-magnetostatic tracking system in [3] includes a transmitting coil positioned at $\left(\tilde{x}^{\mathrm{t}}, \tilde{y}^{\mathrm{t}}, \tilde{z}^{\mathrm{t}}\right)$ with its orientation described by the unit vector $\hat{m}^{\mathrm{t}}$ aligned with its magnetic dipole moment. Equivalently, $\hat{m}^{\mathrm{t}}$ can be defined by the two angles $\theta^{\mathrm{t}}$ and $\varphi^{t}$ in spherical coordinates. The magnetic field of the transmitting coil is measured with a set of $N^{\mathrm{r}}$ identical receiving coils with known locations $\left(\tilde{x}_{k}^{\mathrm{r}}, \tilde{y}_{k}^{\mathrm{r}}, \tilde{z}_{k}^{\mathrm{r}}\right)$ and orientations $\hat{m}_{k}^{\mathrm{r}}$, where $k=1, \ldots, N^{\mathrm{r}}$. The purpose of the system is to estimate $\tilde{\mathbf{p}}=\left[\tilde{x}^{\mathrm{t}}, \tilde{y}^{\mathrm{t}}, \tilde{z}^{\mathrm{t}}, \theta^{\mathrm{t}}, \varphi^{\mathrm{t}}\right]^{T}$, where we assume that the magnitude of each magnetic dipole moment is known. We normalize the spatial coordinates with the distance $h$, which gives $\vec{r}^{\mathrm{t}}=\left(x^{\mathrm{t}}, y^{\mathrm{t}}, z^{\mathrm{t}}\right)=\left(\tilde{x}^{\mathrm{t}} / h, \tilde{y}^{\mathrm{t}} / h, \tilde{z}^{\mathrm{t}} / h\right)$ and $\mathbf{p}=$ $\left[x^{\mathrm{t}}, y^{\mathrm{t}}, z^{\mathrm{t}}, \theta^{\mathrm{t}}, \varphi^{\mathrm{t}}\right]^{T}$. Similarly, the position of the receivers are normalized to be $\vec{r}_{k}^{\mathrm{r}}=\left(x_{k}^{\mathrm{r}}, y_{k}^{\mathrm{r}}, z_{k}^{\mathrm{r}}\right)=\left(\tilde{x}_{k}^{\mathrm{r}} / h, \tilde{y}_{k}^{\mathrm{r}} / h, \tilde{z}_{k}^{\mathrm{r}} / h\right)$.

The induced voltage in the receiving coil $k$ can be expressed as

$$
V_{k}=-j \omega \alpha \frac{\mu_{0}}{4 \pi}\left(\frac{3\left(\hat{m}^{\mathrm{t}} \cdot \vec{R}_{k}\right)\left(\hat{m}_{k}^{\mathrm{r}} \cdot \vec{R}_{k}\right)}{R_{k}^{5}}-\frac{\hat{m}^{\mathrm{t}} \cdot \hat{m}_{k}^{\mathrm{r}}}{R_{k}^{3}}\right)
$$

where $\vec{R}_{k}=\vec{r}_{k}{ }^{\mathrm{r}}-\vec{r}^{\mathrm{t}}$ is the vector of length $R_{k}$ from the transmitting dipole to the receiving dipole. The constant $\alpha$ depends on the coils' characteristics (number of turns, cross-section area, etc.) as well as the current flowing in the transmitting coil. We use $\omega \alpha=4.33 \cdot 10^{6} \mathrm{Am} / \mathrm{s}$ in the tests that follow. Clearly, the induced voltage is non-linear with respect to the position of the transmitter.

Performance metric: Let $V_{k}(\mathbf{p})$ denote the induced voltage in the receiving coil $k$, and let $\nabla_{\mathbf{p}} V_{k}\left(\mathbf{p}_{0}\right)$ denote the gradient of $V_{k}(\mathbf{p})$ with respect to the parameters in $\mathbf{p}$ at the point $\mathbf{p}_{0}$. Under the assumption of additive Gaussian measurement noise that has variance $\sigma^{2}$ and is independent between measurements, the Fisher information matrix

$$
\mathbf{M}=\sum_{k=1}^{N^{\mathrm{r}}} \mathbf{M}_{k}=\sum_{k=1}^{N^{\mathrm{r}}} \frac{\left[\nabla_{\mathbf{p}} V_{k}\left(\mathbf{p}_{0}\right)\right]\left[\nabla_{\mathbf{p}} V_{k}\left(\mathbf{p}_{0}\right)\right]^{T}}{\sigma^{2}}
$$

provides a metric for the performance of the parameter estimation. The Cramér-Rao inequality [6], $\operatorname{cov} \hat{\mathbf{p}} \succeq \mathbf{M}^{-1}$, gives a lower bound for the covariance of the estimate $\hat{\mathbf{p}}$ for an unbiased estimator. Therefore, one way of optimizing the performance of a measurement system is to maximize $\mathbf{M}$ in some sense.

Here, we seek to find the so-called D-optimal sensor positions [6] for the magnetic tracking system given that $\mathbf{p}$ belongs to the measurement domain $\Omega_{\mathbf{p}}$, which is a limited part of the parameter space. Thus, we wish to

$$
\begin{array}{ll}
\underset{\vec{r}_{k}^{r}}{\operatorname{minimize}} & -\log \operatorname{det}\left(\frac{\mathbf{M}\left(\mathbf{p} ; \vec{r}_{1}^{\mathrm{r}}, \ldots, \vec{r}_{N^{\mathrm{r}}}\right)}{N^{\mathrm{r}}}\right) \\
\text { subject to } & \mathbf{p} \in \Omega_{\mathbf{p}}
\end{array}
$$

where we have normalized the cost function with respect to the number of sensors.

Sensor selection and convex relaxation: Following the approach in [5], we express the optimization problem (3) as a sensor selection problem: find which $N^{\mathrm{r}}$ sensors to use $\left(w_{k}=1\right)$ among $K$ candidates. The corresponding mathematical formulation is

$$
\begin{array}{ll}
\underset{w_{k}}{\operatorname{minimize}} & -\log \operatorname{det}\left(\frac{\sum_{k=1}^{K} w_{k} \mathbf{M}_{k}(\mathbf{p})}{N^{\mathrm{r}}}\right) \\
\text { subject to } & \mathbf{p} \in \Omega_{\mathbf{p}} \\
& w_{k} \in\{0,1\}, \quad k=1, \ldots, K \\
& \sum_{k=1}^{K} w_{k}=N^{\mathrm{r}} .
\end{array}
$$

There are $\left(\begin{array}{c}K \\ N^{\mathrm{r}}\end{array}\right)$ combinations wherefore an exhaustive search is tractable only for small problems.

An approximate solution to the sensor selection problem (4) can be obtained as follows. Allow the sensors to perform multiple measurements and let $w_{k}$ denote the number of measurements performed by sensor $k$. The quantity $N^{\mathrm{r}}$ thus corresponds to the total number of measurements. Introduce $\lambda_{k}=w_{k} / N^{\mathrm{r}}$ which is the fraction of the total number of measurements that are performed by sensor $k$. If the total number of measurements is large, a relaxation of the constraint $\lambda_{k} \in \mathbb{Q}$ into $\lambda_{k} \in \mathbb{R}$ can be easily motivated. Thus, we obtain the relaxed problem

$$
\begin{array}{cl}
\underset{\lambda_{k}}{\operatorname{minimize}} & -\log \operatorname{det}\left(\sum_{k=1}^{K} \lambda_{k} \mathbf{M}_{k}(\mathbf{p})\right) \\
\text { subject to } & \mathbf{p} \in \Omega_{\mathbf{p}} \\
& \lambda_{k} \geq 0, \quad k=1, \ldots, K \\
& \sum_{k=1}^{K} \lambda_{k}=1
\end{array}
$$

which is convex [7] and therefore can be readily solved. Furthermore, the relaxed problem provides a lower bound [5] on the optimal value of the cost function for the non-relaxed problem (4). The introduction of the weights $\lambda_{k}$ comes with the benefit of not having to specify the number of sensors $a$ priori. Instead, a threshold $\lambda_{0}$ can be used to investigate how many sensors that should be used.

Results: Consider a planar sensor array in the $x y$-plane with $\hat{m}_{k}^{\mathrm{r}}=$ $(0,0,1) \forall k$. The transmitter is fixed and we choose $h=\tilde{z}^{\mathrm{t}}$, which gives a measurement domain $\Omega_{\mathbf{p}}$ described by the vector $\left[x_{0}, y_{0}, 1, \theta_{0}, \varphi_{0}\right]^{T}$. To solve the relaxed problem (5) for this sensor array and measurement domain, we use CVX, a package for specifying and solving convex programs $[8,9]$. The problems considered in this letter are solved in less than a minute on a conventional desktop computer.

We start by considering the case where the magnetic dipole moment of the transmitter is perpendicular to the sensor plane. Because of the circular symmetry of the magnetic dipole field in the sensor plane, the optimal sensor pattern should also show circular symmetry. We therefore consider a sensor array consisting of $3000 \times 4+1=12001$ sensors uniformly distributed along four radial lines expressed in polar coordinates as $0 \leq$ $\rho \leq 1.5$ and $\phi \in\{0, \pi / 2, \pi, 3 \pi / 2\}$. Here, $\rho$ is the dimension-less radius $\rho=\tilde{\rho} / h$ and the spacing in $\rho$ is $1 / 2000$. The four sensors at a radial distance $\rho$ obtain equal weights. Fig. 1 shows the sum of these weights as a function of $\rho$. For the discretization described above, the largest weights are obtained at $\rho_{1}=0.2665$ and $\rho_{2}=0.9150$. It should be noted that these values do not depend on the number of radial lines with sensors 


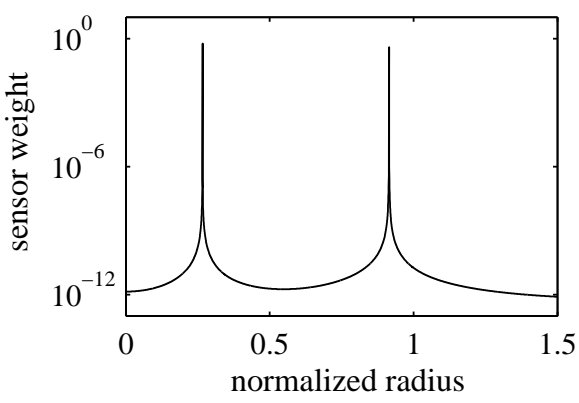

Fig. 1 Sensor weights as a function of the normalized radius $\rho$ for sensors in a polar grid when the magnetic moment of the transmitter is perpendicular to the sensor plane.

as long as it exceeds two. These results should be compared to the values $\rho=0.26649$ and $\rho=0.91540$ that are obtained by an exhaustive search of the corresponding original problem (3), i.e. optimizing the radii of two circles with four sensors each. The cost function value at the optimum is 12.59 for the relaxed problem (5) and 12.69 for the problem (3) with two circles. For comparison, the cost function value is 14.04 for the relaxed problem (5) with uniform weights, i.e. $\lambda_{k}=1 / K \forall k$.

We now turn to the case $\theta \neq 0^{\circ}$ and consider a sensor array that consists of $81 \times 86=6966$ sensors placed on a uniform Cartesian grid with $\left|x^{\mathrm{r}}\right| \leq$ $0.8,\left|y^{\mathrm{r}}\right| \leq 0.85$ and the cell size $1 / 50$. Fig. 2 shows by black areas the sensor positions that yield $\lambda_{k}>\lambda_{0}=10^{-3}$ for $\Omega_{\mathbf{p}}=\left[0,0,1, \theta, 90^{\circ}\right]^{T}$ and different values of $\theta$. The pattern is left-right symmetrical due the same symmetry of the dipole field. The threshold $\lambda_{0}$ was arbitrarily chosen after examining the distribution of $\lambda_{k}$ for a number of test cases. In these test cases, all $\lambda_{k}<\lambda_{0}$ were considerably smaller than $\lambda_{0}$
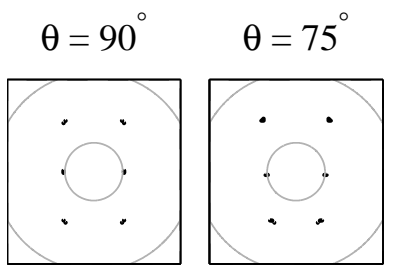

$$
\theta=60^{\circ}
$$

$$
\theta=45^{\circ}
$$

$$
\theta=30^{\circ}
$$
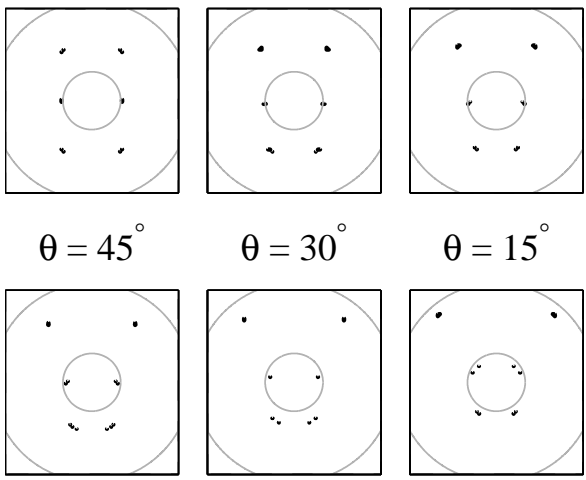

$$
\theta=15^{\circ}
$$

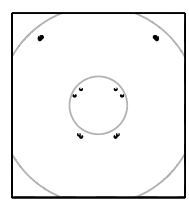

Fig. 2 Contour plot with $\lambda_{k}>\lambda_{0}=10^{-3}$ represented by black areas, for $\Omega_{\mathbf{p}}=\left[0,0,1, \theta, 90^{\circ}\right]^{T}$. The rectangle corresponds to the bounds of the rectangular sensor array. The gray circles correspond to the optimized radii $\rho_{1}=0.2665$ and $\rho_{2}=0.9150$ shown in Fig. 1 for $\theta=0^{\circ}$.

The sensor selection scheme introduces a spatial discretization of the allowed sensor positions. Sensor positions with large weights should therefore be regarded as indicators of favorable regions rather than exact locations for optimal measurements. A translation of the measurement point in the $x$ - and $y$-directions and an azimuthal rotation result in a similar transformation of the weights and sensor positions provided that edge effects are avoided at the bounding edges of the sensor array. If the sensor array is extended by enlarging its dimensions while keeping the cell size constant, the positions of the sensors with $\lambda_{k}>\lambda_{0}$ do not change.

Conclusion: In this letter, we have presented a computationally efficient method for optimizing the sensor positions in a magnetic tracking system. A performance metric based on the Fisher information matrix is exploited to formulate a sensor selection problem. The resulting optimization problem is then relaxed into a convex problem.

The presented results provide basic insights in optimal sensor positions for a measurement domain consisting of one point, which can be exploited during the design of a magnetic tracking system. Furthermore, the proposed method can be used to easily obtain a starting guess for an optimization method that attempts to solve the non-relaxed problem or a problem where the sensor positions are not limited to a set of predetermined positions.

Acknowledgment: Oskar Talcoth has been supported by the Swedish research council VINNOVA via a project within the VINN Excellence center CHASE, and by the Swedish National Graduate School in Scientific Computing.

O. Talcoth and T. Rylander (Dept. of Signals and Systems, Chalmers University of Technology, SE-41296 Göteborg, Sweden)

E-mail: oskar.talcoth@chalmers.se

\section{References}

1 Plotkin, A., Shafrir, O., Paperno, E., and Kaplan, D. M.: 'Magnetic eye tracking: A new approach employing a planar transmitter', IEEE Trans. Biomed. Eng., 2010, 57(5), pp. 1209-1215

2 Biosense Webster: 'Carto 3 System', www. biosensewebster.com/ products / navigation/carto3. aspx, accessed June 29, 2012

3 Iustin, R., Linder, J., Isberg, E., Gustafsson, T., and Lennernäs, B.: 'A Model Based Positioning System', World Intellectual Property Organization, 2008, Patent WO 2008/079071 A1

4 Shafrir, O., Paperno, E., and Plotkin, A.: 'Magnetic Tracking with a Flat Transmitter', (Lambert Academic Publishing, Germany, 2010)

5 Joshi, S., and Boyd, S.: 'Sensor Selection via Convex Optimization', IEEE Trans. Signal Process., 2009, 57(2), pp. 451-462

6 Walter, É., and Pronzato, L.: 'Identification of parametric models from experimental data', (Springer-Verlag, 1997)

7 Boyd, S., and Vandenberghe, L.: 'Convex optimization', (Cambridge University Press, UK, 2004)

8 Grant, M., and Boyd, S.: 'CVX: Matlab Software for Disciplined Convex Programming', version 1.21, April 2011, http: / / cvxr. com/cvx

9 Grant, M., and Boyd, S.: 'Graph implementations for nonsmooth convex programs', in V. Blondel, S. Boyd, and H. Kimura (Eds.): 'Recent Advances in Learning and Control', (Springer-Verlag, 2008), pp. 95-110 